\title{
Commentary: Will cardiac reanimation increase donors for heart transplantation?
}

\author{
Richard C. Daly, MD
}

\author{
From the Department of Cardiovascular Surgery, Mayo Clinic, Rochester, Minn \\ Disclosures: Author has nothing to disclose with regard to commercial support. \\ Received for publication Sept 30, 2019; revisions received Sept 30, 2019; accepted for publication Sept 30, 2019; \\ available ahead of print Jan 22, 2020. \\ Address for reprints: Richard C. Daly, MD, Mayo Clinic, 200 First St SW, Rochester, MN 55905 (E-mail: daly. \\ richard@mayo.edu). \\ J Thorac Cardiovasc Surg 2020;159:e313-4 \\ 0022-5223/\$36.00 \\ Copyright (C) 2019 by The American Association for Thoracic Surgery \\ https://doi.org/10.1016/j.jtcvs.2019.09.144
}

The first human heart transplants were performed prior to a legal definition of brain death and, thus, required donation of the heart after circulatory death was declared, followed by reanimation of the heart in the recipient. Cardiac donation after circulatory death (DCD) has been evaluated in animal models for at least 20 years, initially contemplating cardiac reanimation in trauma victims. ${ }^{1}$ The establishment of DCD protocols for abdominal organs and lungs has allowed more deliberate and controlled circumstances but still involves a warm ischemic time before organs are flushed and cooled and also requires cessation of cardiac activity without cardiac protection; therefore, consideration of DCD for heart transplantation has been delayed.

Admirably, Jawitz and colleagues ${ }^{2}$ call attention to the potential for using DCD donors for heart transplantation in the United States and have tried to estimate the eventual maximum potential number of cardiac DCD donors in the United States based on United Network for Organ Sharing data of lung and abdominal DCD donors, which is an inherently optimistic effort. The authors have provided reasonable justification of their estimate as well as recognizing the limitations in their study. It is important to understand the method used because accurate estimate permits responsible allocation of resources for the time, research funding, potential industry or private investment, and the eventual practice changes involved.

Estimating potential DCD heart donors depends on what criteria define an acceptable donor. There are 2 reports of DCD for clinical heart transplantation, each with different criteria for selecting a cardiac DCD donor. Based on donor criteria in the UK study, ${ }^{3}$ the authors estimate an increase in the number of heart transplants in the United States by $30 \%$ per year in the United States. Criteria for DCD donors were stricter in the Australian report and predict DCD donors would increase heart transplants in the United States by $21 \%$ per year. ${ }^{4}$ Recognizing that actual donors used did not reach the limits of the criteria in these studies and that the DCD donors in the United Network for Organ

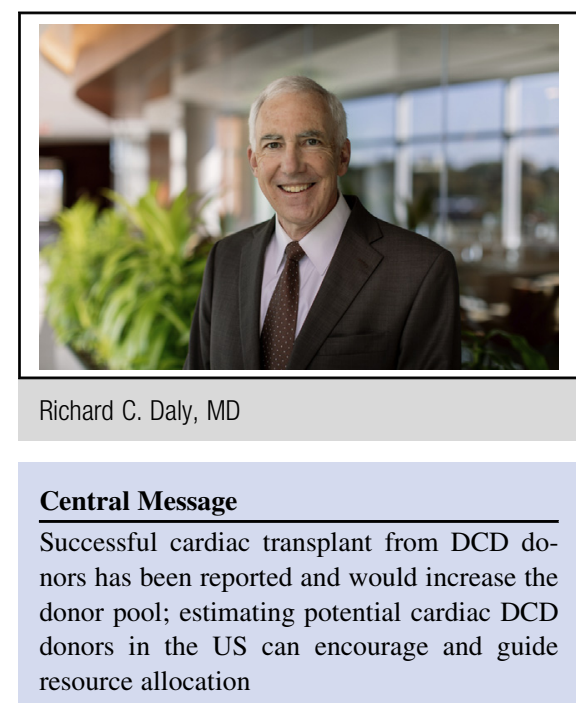

See Article page e307.

Sharing database were not evaluated as heart donors (so no echocardiograms and limited documentation of inotropes), the authors estimate use of DCD donors would potentially increase heart transplants in the United States by $10 \%$ to $20 \%$ per year.

There is also the issue of dissemination of DCD donors into actual practice. Lung transplant experience indicates that actual use of DCD donors will have a gradual incorporation into the practice. The number of DCD donors for lung transplantation quadrupled from 2012 to 2017, yet they still accounted for only $3.6 \%$ of lung transplants in 2017.

Although it is appropriate to understand the limitations of the estimates of changes in transplant volumes, the efforts to use DCD donors for heart transplantation may also lead to an increase in heart transplants from traditional donation after brain death donors due to promotion of investment in ex vivo perfusion and other technologies that might lead to expansion of the donation after brain death donor pool and use of more extended-criteria donors.

\section{References}

1. Ferrera R, Marcsek P, Guidollet J, Berthet C, Dureau G. Lack of successful reanimation of pig hearts harvested more than 10 minutes after death. J Heart Lung Transplant. 1994;14:322-8.

2. Jawitz OK, Raman V, DeVore AD, Mentz J, Patel CB, Rogers J, et al. Increasing the United States heart transplant donor pool with donation after circulatory death. J Thorac Cardiovasc Surg. 2020;159:e307-9.

3. Messer S, Page A, Axell R, Berman M, Hernandez-Sanchez J, Colah S, et al Outcome after heart transplantation from donation after circulatory-determined death donors. J Heart Lung Transplant. 2017;36:1311-8. 
4. Chew HC, Iyer A, Connellan M, Scheuer S, Villanueva J, Gao L, et al. Outcomes after circulatory death heart transplantation in Australia. J Am Coll Cardiol. 2019; $73: 1447-59$.
5. OPTN/SRTR 2017 Annual Data Report: Lung, Table LU 8. Available at: https:// srtr.transplant.hrsa.gov/annual_reports/2017/Lung.aspx\#LU_8_char_adult_tx_ tx. Accessed September 16, 2019. 